\section{Effectiveness and tolerability of tapentadol in very elderly patients with assessment of cognitive-behavioral aspects}

Eleonora Nunziata, ${ }^{1}$ Francesco De Siati,
Lorenzo ${ }^{2}$ Palleschi ${ }^{2}$

${ }^{1}$ Department of Emergency; and ${ }^{2}$ Unit of Geriatrics, AO S. Giovanni-Addolorata, Rome, Italy

\begin{abstract}
Pain is a common complaint of the elderly and the prevalence of persistent discomfort increases with aging. Suffering may be underreported as some elderly patients incorrectly believe that pain is a normal process of aging. The aim of this study is to assess the analgesic effectiveness and tolerability of tapentadol in elderly patients with assessment of cognitivebehavioral aspects. During treatment with tapentadol, elderly patients experienced reduced pain intensity and improved physical and mental health.
\end{abstract}

\section{Introduction}

The prevalence of pain increases with each decade of life but pain, though frequent, is not part of physiological ageing. ${ }^{1}$ In Italy persistent pain, or pain that lasts more than three months, is related to progressive nonneoplastic pathologies, affects from $40 \%$ to $85 \%$ of the very elderly population, and $2 / 3$ of cases are caused by musculoskeletal diseases. $^{2,3}$ About $60 \%$ of older people in the country, and $80 \%$ of those residing in long term care facilities, suffer from pain. ${ }^{3}$

Managing pain in the elderly is not an easy challenge. ${ }^{4}$ Cure is all the more effective when it takes into account the fragility of these subjects due to a large number of factors, including physical health, cognitive state, psycho-emotional state, functional state, socio-economic condition and lifestyle. ${ }^{4,5}$ Further complicating the therapeutic choice is the fact that elderly patients are less tolerant of analgesics, anti-inflammatory drugs, and some adjuvant agents such as tricyclic antidepressants, and the way in which age affects various parameters of drug absorption and elimination, which varies from one subject to another. ${ }^{5,6}$

Tapentadol possesses an original and innovative mechanism of action: an agonist of opioid receptors $\mu$ (MOR) and noradren- aline reuptake inhibitor (NRI); both mechanisms of action contribute complementarily and synergically to its analgesic efficacy, demonstrated in various models of both nociceptive and neuropathic pain. ${ }^{7,8}$ Furthermore, tapentadol is very safe in relation to the low risk of drug interactions due to reduced plasma protein binding, lack of impact on CYP450 enzymes, the major metabolic pathway through glucuronidation, and the absence of active metabolites. ${ }^{8}$

\section{Aim}

The aim of the study was to assess the analgesic effectiveness and tolerability of tapentadol PR (50-250 mg BID) in very elderly patients, with assessment of cognitivebehavioral aspects.

\section{Materials and Methods}

Forty patients were enrolled (mean age 78.9 years, SD 5.7, 88\% women), with pain intensity greater than or equal to 4 on the numerical rating scale (NRS).

Analgesic therapy using tapentadol PR was begun at a dose of $50 \mathrm{mg}$ twice a day (BID), increased if necessary to $50 \mathrm{mg}$ twice a day up to total daily dose not exceeding $500 \mathrm{mg}$. The patients were examined upon joining the study (V0), and after 7 (V1), 30 (V2) and 60 days (V3).

\section{Primary endpoints}

Percentage of responder patients, i.e. patients with a reduction of pain intensity of at least $30 \%$, at the end of observation, compared to baseline, evaluated using NRS from 0 to 10 .

\section{Secondary endpoints}

- Assessment of quality of life using the short form-12 questionnaire (SF-12).

Assessment of cognitive status and functional autonomy was made using the mini-mental state examination questionnaire (MMSE), the BADL questionnaire (basic activity of daily living) and IADL (instrumental activity of daily living).

- Adherence to therapy evaluated by dropout number.

\section{Tolerance assessment}

During the observation period all side effects were recorded, specifically for their severity, duration and possible therapeutic measures.

\section{Analysis of the main variable}

The main variable, patient responder frequency, was analyzed by evaluating the
Correspondence: Eleonora Nunziata,

Department of Emergency, AO S. Giovanni Addolorata, Rome, Italy.

E-mail: ele.nunziata@gmail.com

Key words: Tapentadol; pain; elderly.

Received for publication: 11 July 2017. Accepted for publication: 11 July 2017.

This work is licensed under a Creative Commons Attribution-NonCommercial 4.0 International License (CC BY-NC 4.0).

(C) Copyright E. Nunziata et al., 2017

Licensee PAGEPress, Italy

Geriatric Care 2017; 3:6900

doi:10.4081/gc.2017.6900

number of responders compared to the actual number of patients admitted to the study. $95 \%$ confidence intervals were reported.

This assessment also applies to those dropout patients for whom an NRS scale is available beyond the baseline; to calculate the reduction percentage the last available survey was used.

\section{Analysis on other clinical parameters}

Intensity of pain, detected at intervals called for during the study period, was assessed by analyzing variance for repeated measurements without grouping with multiple comparisons versus baseline. The quality of life (questionnaire SF-12) was evaluated by test $t$ for paired samples by comparing the value found at the end of the study with the baseline. Finally, cognitive-behavioral questionnaires (BADL, IADL and MMSE) were evaluated using the Wilcoxon test; also in this case a comparison was performed of the final value with the baseline.

Adverse events were reported in a descriptive manner.

The threshold value used to evaluate the significance of statistical tests is $0.05(5 \%)$.

\section{Results}

Within the first 60 days (visit V3) of treatment with tapentadol, 28 patients out of $39(72 \%)$ were responders to therapy (95\%: CI $55 \%-85 \%)$. In the period between the initial visit and the one after 7 days, the intensity of pain diminished by $22 \%$, down from an average value of 7.2 on the NRS scale to an average of 5.6. After 30 days, it decreased by $39 \%(\mathrm{NRS}=4.4)$ while, after 60 days, the reduction was $58 \%$ (NRS = 3.0). The reduction in pain intensity was 


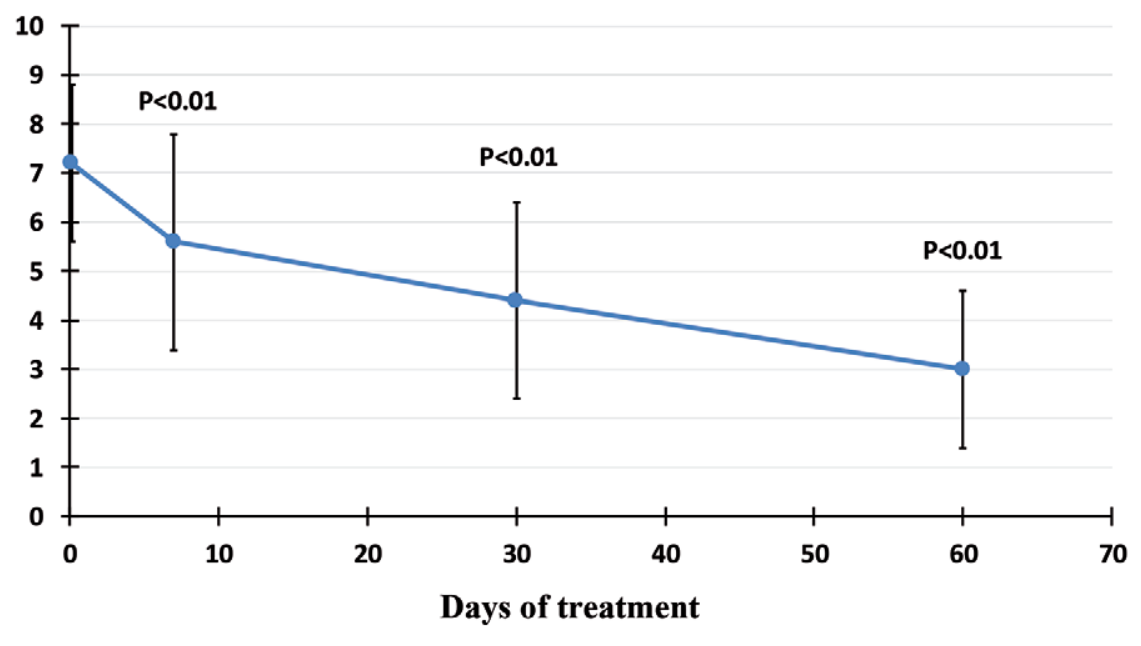

Figure 1. Pain intensity, median $\pm \mathrm{SD}(\mathrm{N}=27)$.

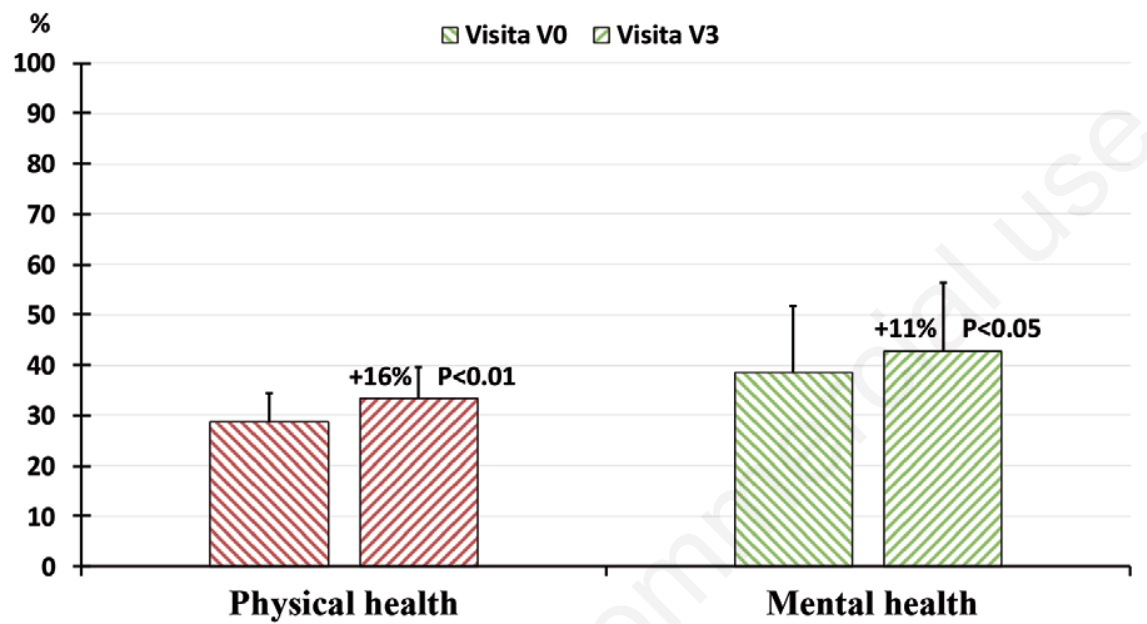

Figure 2. Short form-12 questionnaire median $\pm \mathrm{SD}(\mathrm{N}=23)$.

statistically significant for all follow-up visits $(\mathrm{P}<0.01)$ (Figure 1).

Physical and mental health improved significantly $(\mathrm{P}<0.01$ for physical health and $\mathrm{P}<0.05$ for mental health) (Figure 2).

No significant changes in functional autonomy and cognitive status were detected during follow-up.

Before the end of the study, 13 out of 40 patients $(32 \%)$ discontinued treatment with tapentadol; seven suspensions (18\%)
20 side effects were reported by 12 patients $(30 \%)$ : the most frequent were headache, drowsiness, mental confusion and dizziness.

\section{Conclusions}

The analysis shows pain in elderly patients was reduced by administering tapentadol. The reduction in pain intensity was associated with a significant improvement in the physical and mental health.

\section{References}

1. Abdulla A, Adams N, Bone M. Guidance on the management of pain in older people. Age Ageing 2013;42:1-57.

2. Smith AK, Cenzer IS, Knight SJ. The epidemiology of pain in the last 2 years of life. Ann Intern Med 2010;153:563-9.

3. Patel KV, Guralnik JM, Dansie EJ, Turk DC. Prevalence and impact of pain among older adults in the United States: findings from the 2011 National Health and Aging Trends Study. Pain 2013; 154:2649-57.

4. Kaye AD, Baluch A. Pain management in the elderly population: a review. Ochsner J 2010;179-87.

5. Reid MC, Eccleston C, Pillemer K. Management of chronic pain in older adults. BMJ 2015;350:h532.

6. Rastogi R, Meek BD. Management of chronic pain in elderly, frail patients: finding a suitable, personalized method of control. Clin Interv Aging 2013;8:37-46.

7. American Geriatrics Society Panel on Persistent Pain in Older Persons. Pharmacological management of persistent pain in older persons. J Am Geriatr Soc 2009;57:1331-46.

8. Michael A, Gerhard HH, MuellerSchwefe. Efficacy and tolerability balance of oxycodone/naloxone and tapentadol in chronic low back pain with a neuropathic component: a blinded end point analysis of randomly selected routine data from 12-week prospective open-label observations. J Pain Res 2016;9:1001-20. 